\section{Synchronized defibrillation for ventricular fibrillation}

\author{
Karen M Darragh', Ganesh Manoharan', Cesar Navarro', \\ Simon J Walsh', John D Allen², John McC Anderson ${ }^{3}$ and \\ AA Jennifer Adgey'
}

European Heart Journal: Acute Cardiovascular Care I (4) 285-290

(C) The European Society of Cardiology 2012

Reprints and permission:

sagepub.co.uk/journalsPermissions.nav

DOI: 10.1 1 $77 / 2048872612458498$

acc.sagepub.com

@SAGE

\begin{abstract}
Objective: Optimization of defibrillation success is important to improve efficacy and minimize post-shock sequelae. Previous work has suggested an improvement in shock success when an intracardiac shock is delivered synchronized to the upslope of a VF wave. We investigated the efficacy of transthoracic defibrillation success using a novel external biphasic defibrillator which delivers shocks synchronized to the upslope of the surface ECG.

Methods: A prospective, controlled, randomized study in a research institute laboratory of male and female pigs $(54.2 \pm 1.8 \mathrm{~kg})$. Ventricular fibrillation (VF) was induced in 10 anaesthetized and ventilated pigs. Shocks were delivered randomly from a biphasic defibrillator in synchronized or non-synchronized mode via self-adhesive electrode pads following $30 \mathrm{~s}$ of VF. Energy settings at 50, 70, 80, and I00 J were randomly tested. VF amplitude, impedance, and shock outcome were recorded and analysed digitally.

Results: A total of 300 shocks were delivered. Synchronized shocks were delivered on the upslope of the VF wave in $99 \%$ of cases. There was no significant difference in shock success between shocks delivered in synchronized or non-synchronized modes $(p=0.695)$. There was no significant difference in the amplitude of VF between successful and unsuccessful shocks $(p=0.163)$. Furthermore, there was no association between shock success and transthoracic impedance.
\end{abstract}

Conclusion: The novel defibrillator used in this study was able to consistently deliver shocks on the upslope portion of the VF wave but did not show an improvement in shock success.

\title{
Keywords
}

Electric defibrillation, electrocardiogram, external defibrillators, impedance, swine, ventricular fibrillation

Received: 6 May 2012; accepted: 27 July 2012

\section{Introduction}

Over the last number of years, changes to the resuscitation guidelines have shifted the focus of resuscitation towards the delivery of good-quality cardiopulmonary resuscitation (CPR). The traditional stacked shocks protocol has been altered in favour of single shocks interspersed by longer periods of CPR. ${ }^{1}$ Currently available defibrillators deliver shocks randomly throughout the VF cycle with shock delivery occurring immediately once the device is fully charged. Frequency analysis of ventricular fibrillation (VF) has suggested a dominant frequency exists which may signify a degree of organization of VF. ${ }^{2,3}$ Studies have since demonstrated improvements in shock success when defibrillating shocks were timed to various parameters on the electrocardiogram (ECG). ${ }^{4-8}$ No study as yet has demonstrated a significant improvement in shock success when synchronized shocks for VF are delivered transthoracically.

\footnotetext{
'Belfast Health and Social Care Trust, Belfast, UK.

${ }^{2}$ Queen's University, Belfast, UK.

3 University of Ulster at Jordanstown, Newtownabbey, UK.
}

\section{Corresponding author:}

Karen M Darragh, The Heart Centre, Belfast Health and Social Care Trust, Grosvenor Road, Belfast, BTI2 6BA, Northern Ireland, UK.

Email: karendarragh@yahoo.co.uk 


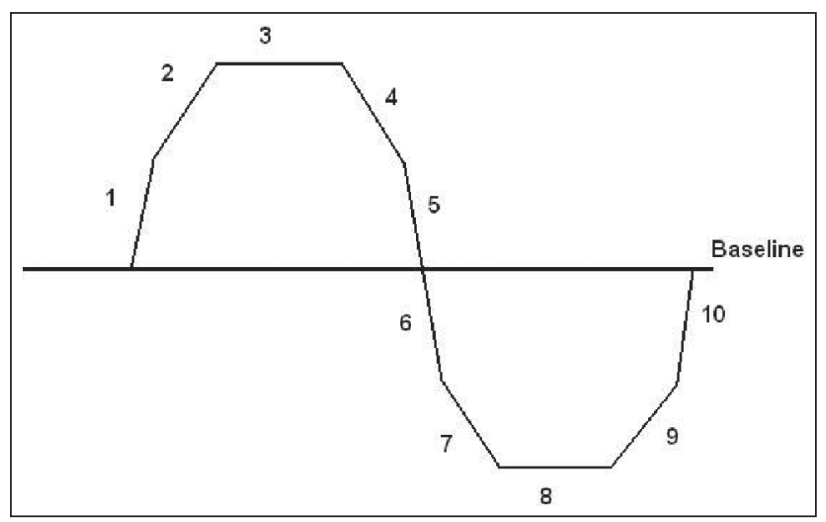

Figure I. Line drawing depicting theoretical site positions on ventricular fibrillation wave

The purpose of our study was to assess the effect of synchronizing shocks to the upslope of the surface ECG for transthoracic defibrillation.

\section{Materials and methods}

\section{Device development}

A novel biphasic defibrillator was developed capable of ECG analysis and synchronization signalling which allowed a programmable energy to be delivered at different points during the VF cycle (Figure 1). From the surface ECG (using self-adhesive pads), the most positive and negative voltages could be measured allowing detection of the peak and trough amplitude of the VF waveform. The device calculates the average minimum and maximum amplitudes of the VF waves over a moving 4-s window. A defibrillating shock will be delivered at a pre-specified percentage level of these average amplitudes. For example, if the percentage level is set at $80 \%$, a shock will be delivered when the device next detects a VF wave which has both a minimum and maximum amplitude that is at least $80 \%$ of this 4-s average. Preliminary experiments in our laboratory investigated varying percentage levels $(20,40,60,80$, $100 \%$ ) of the 4 -s average amplitudes. These experiments demonstrated the $80 \%$ level to be the most consistent level at delivering the shock on the upslope of the VF wave. This setting was therefore used for the following study.

By meeting both the minimum and maximum amplitude criteria, the device also aimed to deliver shocks during higher-amplitude VF as some previous work had suggested that higher-amplitude VF would be more susceptible to defibrillation. ${ }^{9}$ Once a suitable VF wave has been detected, a trigger signal is sent (instantaneously) to the defibrillator resulting in shock delivery within $1 \mathrm{~ms}$ of the detected ECG event. The device was also capable of delivering non-synchronized shocks by disabling the synchronization function. In this mode, shocks were delivered randomly throughout the VF cycle once the device had charged.

\section{Animal preparation}

All work was carried out in accordance with the United Kingdom Animals (Scientific Procedures) Act of 1986.

This study used 10 domestic swine from a registered breeding establishment. Each animal was sedated initially with an intramuscular injection of $1-2 \mathrm{mg} / \mathrm{kg}$ azaperone (Stresnil, 40 mg/ml; Janssen Animal Health, Bucks, UK), followed by an intravenous dose of $20-30 \mathrm{mg} / \mathrm{kg}$ sodium pentobarbital (Sagatal, $60 \mathrm{mg} / \mathrm{ml}$; Rhône Mérieux, Harlow, UK) via peripheral access. Each animal was then intubated and maintained in the dorsal recumbent position and ventilated with room air (model 16/24, Ideal Respirator; CF Palmer, London, UK). Additional sodium pentobarbital was used throughout the experiment to maintain anaesthesia.

Heart rate, lead II ECG, arterial blood pressure, capillary oxygen saturation, and end-tidal carbon dioxide concentration were monitored continuously throughout the procedure to ensure maintenance within normal physiological limits. Rectal temperature was measured half hourly and body temperature was maintained using a heated blanket (Homeothermic Blanket Control Unit; Harvard Apparatus, Kent, UK). Arterial blood gas and electrolyte measurements were made at the beginning, middle, and end of each experiment to ensure consistency between the animals.

Central access was obtained via the left external jugular vein (8 French sheath; Cordis) and the left internal carotid artery (7 French sheath; Cordis). A pacing catheter for induction of VF was placed in the apex of the right ventricle under fluoroscopic control (Siemens).

Adhesive electrode pads (Samaritan AED Pads; HeartSine Technologies) were applied to the shaved thoracic surface in the right parasternal position and in the left lateral position with the inferior border over the apex. Each set of pads was changed after approximately 20 uses to ensure good contact with the skin.

\section{Protocol}

VF was induced by rapid stimulation of the right ventricle $(100 \mathrm{~Hz} ; 5 \mathrm{~ms}$ stimuli; 10V; for $5 \mathrm{~s}$ ) via a stimulator (Grass S44; Quincey, MA, USA). Once VF was present for $30 \mathrm{~s}$, a defibrillating shock was delivered.

Shocks were delivered in either synchronized or nonsynchronized mode at $50,70,80$ or $100 \mathrm{~J}$ using a Latin square method. Each energy setting was evaluated four times in each experiment in random order (in both synchronized and non-synchronized modes) resulting in a 32-shock protocol. ECG data were recorded on customized software (Notocord version 0.08, 2005 edition) to allow calculation of delays in shock delivery. A shock was considered successful when VF was removed for $\geq 5 \mathrm{~s}$. Following any failed shock, rescue shocks were delivered from the Codemaster Monophasic defibrillator at $200 \mathrm{~J}$ (up to $360 \mathrm{~J}$ 
Table I. Shock delivery site for synchronized and nonsynchronized shocks.

\begin{tabular}{|c|c|c|c|}
\hline VF wave position & Synchronized & Non-synchronized & Total \\
\hline I & $23(15.3)$ & $9(6)$ & $32(10.7)$ \\
\hline 2 & II $3(75.3)$ & $16(10.7)$ & $129(43)$ \\
\hline 3 & $12(8)$ & $33(22)$ & $45(15)$ \\
\hline 4 & 0 & $19(12.7)$ & $19(6.3)$ \\
\hline 5 & 0 & $14(9.3)$ & $14(4.7)$ \\
\hline 6 & 0 & $7(4.7)$ & $7(2.3)$ \\
\hline 7 & 0 & 7 (4.7) & $7(2.3)$ \\
\hline 8 & $\mathrm{I}(0.7)$ & $28(18.7)$ & $29(9.7)$ \\
\hline 9 & I (0.7) & $13(8.7)$ & 14 (4.7) \\
\hline 10 & 0 & $4(2.7)$ & $4(1.3)$ \\
\hline Total & 150 & 150 & 300 \\
\hline
\end{tabular}

Values are $n(\%)$. VF wave positions are illustrated in Figure I.

Table 2. Shock success and transthoracic impedance for synchronized and non-synchronized modes.

\begin{tabular}{lllll}
\hline Mode & Total shocks & Successful shocks & p-value & Transthoracic impedance $(\Omega)$ \\
\hline Synchronized & 150 & $38(25)$ & 0.695 & $36.63 \pm 5.73$ \\
Non-synchronized & 150 & $42(28)$ & & $36.38 \pm 5.25$ \\
\hline
\end{tabular}

Values are $n, n(\%)$ or mean \pm SD.

as required) via the same electrode pads. A recovery period of at least 3 min was allowed following a successful shock to restore baseline haemodynamics with at least $5 \mathrm{~min}$ recovery after a rescue shock.

Data for each shock were stored on a digital oscilloscope from a voltage probe (custom built). Data from the rescue shocks were not recorded as these shocks had to be delivered without delay and the prolonged oscilloscope processing times would have precluded this. Only the shocks delivered by the study defibrillator were analysed. These data were analysed to determine the point of contact of the shock with the VF wave. Upon completion of the protocol, the animal was euthanized using sodium pentobarbital.

\section{Statistics}

SPSS version 17 (SPSS, Chicago, IL, USA) was used for all statistical analyses. Differences in shock success between synchronized and non-synchronized modes were compared using Chi-squared test. A $p$-value of $<0.05$ was considered statistically significant. The t-test or non-parametric tests were used to assess continuous variables. Values are expressed as mean \pm standard deviation.

\section{Results}

A total of 300 shocks were delivered to 10 swine $(54.2 \pm 1.8 \mathrm{~kg})$ from the study defibrillator during this study. In addition, 252 rescue shocks were required. The rescue shocks are not included in any subsequent analyses. Failure to complete the 32 -shock protocol occurred in two animals. In one case this was due to the development of a non-shockable pulseless rhythm during a series of non-synchronized shocks and in the other case due to dislodgement of the endotracheal tube with failure to reintubate. These animals received 30 and 14 shocks, respectively.

The device successfully delivered shocks on the upslope of the VF wave (positions 1-3, Figure 1.) whilst in synchronized mode (99\%). During non-synchronized mode, shocks fell by chance on the upslope of the VF wave in just over one-third of cases (39\%). Overall, 69\% of all delivered shocks fell on the upslope of the VF wave. A breakdown of shock delivery sites for all shocks is shown in Table 1.

Shock success for both synchronized and non-synchronized modes are shown in Tables 2 and 3. The percentages of shock success rose with increasing energy levels. There was no significant difference between shock success rates for synchronized or non-synchronized modes regardless of energy level $(p=0.695)$. The difference in shock success for any shock delivered on the upslope (either in synchronized or non-synchronized mode) compared to shocks delivered elsewhere was also not significant (upslope 54/206; elsewhere 26/94; $p=0.79$ ).

Analysis of shock success dependent on the length of the protocol revealed no significant difference. There was no increase in shock success for shocks delivered within the first $30 \mathrm{~min}$ of the protocol compared to those delivered in the final $30 \mathrm{~min}(p=0.18)$.

No transthoracic impedance data were available for 14 shocks due to an equipment problem; therefore, the 
Table 3. Shock success for each energy level in synchronized and non-synchronized modes.

\begin{tabular}{llcccc}
\hline Shock energy $(J)$ & $\begin{array}{l}\text { Total shocks } \\
\text { delivered }\end{array}$ & $\begin{array}{l}\text { Successful } \\
\text { shocks }\end{array}$ & $\begin{array}{l}\text { Success in synchronized } \\
\text { mode (\%) }\end{array}$ & $\begin{array}{l}\text { Success in non-synchronized } \\
\text { mode (\%) }\end{array}$ & $p$-value \\
\hline 50 & 74 & $\mathrm{I}(1)$ & $0 / 37(0)$ & $1 / 37(3)$ & 1.0 \\
70 & 75 & $7(9)$ & $1 / 37(3)$ & $6 / 38(16)$ & 0.108 \\
80 & 76 & $24(32)$ & $13 / 38(34)$ & $11 / 38(29)$ & 0.622 \\
100 & 75 & $48(64)$ & $24 / 38(63)$ & $24 / 37(65)$ & 0.878 \\
\hline
\end{tabular}

Values are $n, n(\%)$ or $n /$ total (\%).

Table 4. Shock delivery delay compared with shock success.

\begin{tabular}{lllll}
\hline Shock outcome & \multicolumn{3}{l}{ Delay to shock delivery (quartiles) } \\
\cline { 2 - 5 } & Ist $(0.8-1.8 \mathrm{~s})$ & 2nd $(1.9-2.3 \mathrm{~s})$ & 3rd $(2.4-3.2 \mathrm{~s})$ & 4th $(3.3-14.6 \mathrm{~s})$ \\
\hline Failure & $44(60.3)$ & $54(73.0)$ & $61(78.2)$ & $61(81.3)$ \\
Success $^{\text {p-value }}{ }^{\mathrm{a}}$ & $29(39.7)$ & $20(27.0)$ & $17(21.8)$ & $14(18.7)$ \\
\hline
\end{tabular}

ap-values are for comparison to Ist timing quartile for shock success

following calculation is based on 286 shocks. For all shocks, the impedance averaged $36.5 \pm 5.5 \Omega$ and did not differ significantly between the synchronized and non-synchronized shocks (Table 2). There was no significant difference in impedance between successful and unsuccessful shocks ( $36.58 \pm 4.04$ vs. $36.48 \pm 5.91 \Omega$, respectively).

The time from device charging to shock delivery was analysed for both synchronized and non-synchronized groups (from button pressed to defibrillate to time of delivery on waveform). Synchronized shocks were delivered more slowly than non-synchronized shocks (median $3.0 \mathrm{~s}$, IQR 2.0-4.5 vs. $2.1 \mathrm{~s}$, IQR 1.7-2.6, respectively) (both successful and unsuccessful shocks). Dividing shocks into quartiles dependent on shock delivery delay and shock outcome revealed a difference in shock success between shocks delivered early and those delivered later, becoming more significant as time delay increases (Table 4).

The amplitude of the VF waves was analysed using the root mean square (RMS) method. The RMS amplitude over a window of $6 \mathrm{~s}$ was calculated and plotted over time during VF. There was no significant difference between successful and unsuccessful shocks although there was a trend towards a higher RMS in the successful shocks group (mean $_{\mathrm{RMS}} \pm \mathrm{SD}$ for successful shocks $9.51 \pm 1.13 \mathrm{~V}_{\mathrm{RMS}}$, failed shocks $9.28 \pm 1.36 \mathrm{~V}_{\mathrm{RMS}} ; p=0.163$ ).

\section{Discussion}

Varying features of ventricular fibrillation such as the variation between coarse and fine VF and the variability of shock success at varying energy levels have prompted numerous experiments whose aims were to determine the most opportune time to deliver a defibrillating shock. Early work using frequency analysis and more recently mapping techniques have suggested some degree of organization within VF due to the presence of a dominant frequency. ${ }^{2,3}$ This suggestion that VF is not an entirely random arrhythmia has led researchers to believe that there may exist an optimal time in a VF wave at which a defibrillating shock should be delivered to increase the likelihood of success. Previous work studying the association between defibrillation success and the absolute VF voltage in an animal model has demonstrated an improvement in shock success when shocks were delivered at instants of higher absolute VF voltages. ${ }^{10}$ Other workers have since suggested that the findings of this study may have been more related to the slope of the waveform rather than the amplitude as during these small time-averaging windows which closely follow the electrogram morphology, the shock was likely to have been delivered on the upslope. ${ }^{5}$ Further work has demonstrated a significant improvement in shock success and a reduction in delivered energy with shocks timed to the upslope of the VF wave as opposed to the downslope. ${ }^{6,7}$ This benefit was seen only when shocks were timed to the endocardial (morphology) electrogram and not the surface ECG leads. ${ }^{7}$ It has been postulated that this occurred because the regions in the ventricle which contribute most to the electrogram signal would be those that are in the highest current-density regions during the shock and the location of the upslope of the VF waveform in this lead would be moving in the optimum direction for defibrillation. Defibrillating shocks delivered at this time would therefore extend the refractory period of those cells in the high-density region late in their refractory period while the cells in the low-density region early in the action potential 
would have a long-enough refractory period to halt further propagation of wavefronts in these areas. ${ }^{6,7} \mathrm{~A}$ small but significant reduction in defibrillation threshold energy was noted in a further study when rounded biphasic shocks were delivered timed to the peak and trough of the VF wave as compared to non-synchronized shocks. ${ }^{11}$ Despite these earlier findings, we were not able to demonstrate similar results despite significant advancements in equipment function.

The novel defibrillator developed for this study worked well and achieved excellent results in terms of shock delivery site with $99 \%$ of synchronized shocks delivered on the upslope of the VF wave. Interestingly, in this study, nonsynchronized (or random) shocks most commonly fell on the upslope of the VF wave in $39 \%$ of shocks. This resulted in $69 \%$ of all shocks (i.e. synchronized and non-synchronized) delivered on the upslope of the VF wave. However, this did not translate into an overall benefit. These results show that timing defibrillating shocks to the upslope of the VF waveform on the surface ECG did not result in an improvement in shock success. As mentioned previously, the use of surface ECG leads for shock synchronization may be one factor contributing to the lack of benefit seen during this study.

Overall shock success was not significantly different between the two groups (synchronized vs. non-synchronized, $p=0.695$ ). As would be expected, the likelihood of successful defibrillation increased with increasing energy level in both the synchronized and non-synchronized groups. On further breakdown, there was no significant difference between synchronized and non-synchronized shocks regardless of shock energy.

The VF wave was divided into 10 separate areas (Figure 1). None of these 10 areas as detected on the surface ECG were seen to correspond to an improvement in defibrillation success during this study ( $p>0.05$ between all sites). Small numbers of shocks were delivered to at least three of the 10 shock positions, meaning that interpretation of this result is guarded.

The association between reduced impedance and improved shock success has been demonstrated in a number of experiments utilizing monophasic waveforms. ${ }^{12,13}$ This is potentially due to the change in waveform shape associated with higher impedances..$^{14}$ However, we found no difference in transthoracic impedance between successful and unsuccessful shocks.

Given that early shock delivery is key in short-duration $\mathrm{VF}$, minimizing any delays in shock delivery is of significant importance. Given the irregular pattern of VF, the duration of shock delivery delay will ultimately vary between VF episodes. The delays described in this study are not a reflection of a technical limitation of the device. Given that pre-specified ECG parameters are set for shock delivery, these criteria must be met before the device will attempt to deliver the shock. During this study, we did not analyse the delay from detection of an appropriate wave on which to deliver a shock until the shock was actually delivered. This had previously been extensively tested by the engineers with excellent results. Delay times quoted represent the time between pressing the shock button on the device and the device delivering the shock. During this study, the delays in shock delivery were shown to be associated with a reduction in shock success which became more marked as the delay increased (Table 4). There was only a small difference in average shock delays between the two groups (synchronized vs. non-synchronized), which is unlikely to exert a significant influence on shock outcome. Previous human studies have shown a significant reduction $(10 \%)$ in successful outcomes for every minute that defibrillation is delayed from the time of collapse..$^{15}$ The differences in delay times in our study do not reach $1 \mathrm{~min}$ but the trend in worsening outcomes with increasing delay reflects this same pattern.

Earlier synchronization work by our group encountered delays of up to $8 \mathrm{~s}$ for shock delivery timed to the peak or trough of the VF wave. ${ }^{11}$ In spite of these delays, the results from this study confirmed a small reduction in energy requirements with synchronization, suggesting that our timing delays, which were considerably shorter, were not the only reason for a lack of benefit with synchronization.

Utilization of an $80 \%$ threshold level for the synchronization of defibrillating shocks was intended to result in shock delivery on the upslope of the VF wave. This threshold setting was also intended to result in shock delivery on higher-amplitude VF waves in order to maximize the chance of defibrillation success. As mentioned above, previous work has suggested that higher-amplitude VF may be associated with an improvement in shock success ${ }^{9}$ although other investigations have not confirmed this association. ${ }^{5}$ In keeping with this earlier work, the data obtained from this study did not demonstrate any difference in VF amplitude between successful and unsuccessful shocks.

\section{Conclusion}

The novel defibrillator used during this study successfully synchronized defibrillating shocks to the upslope portion of the VF wave on the surface ECG. Timing delays were also shortened in comparison to previous studies. Despite these factors, shock synchronization in this animal model failed to improve defibrillation success in short-duration VF. Given these results, it seems likely that the upslope of the VF wave on the surface ECG does not correspond to the repolarization of cells that could augment the likelihood of defibrillation success.

\section{Funding}

This work was supported by the Heart Trust Fund, Royal Victoria Hospital, Belfast (grant RG000310 to KMD). 


\section{References}

1. Deakin CD, Nolan JP, Soar J, et al. European Resuscitation Council Guidelines for Resuscitation 2010. Section 4. Adult advanced life support. Resuscitation 2010; 81: 1305-1352.

2. Herbschleb JN, Heethaar RM, van der Tweel I, et al. Signal analysis of ventricular fibrillation. Computers in cardiology. IEEE Computer Society, 1979, p. 49-54.

3. Carlisle EJ, Allen JD, Bailey A, et al. Fourier analysis of ventricular fibrillation and synchronisation of DC countershocks in defibrillation. J Electrocardiol 1988; 21: 337-343.

4. Hsia PW, Frerk S, Allen CA, et al. A critical period of ventricular fibrillation more susceptible to defibrillation. Pacing Clin Electrophysiol 1996; 19: 418-430.

5. Hsu W, Lin Y, Heil JE, et al. Effect of shock timing on defibrillation success. Pacing Clin Electrophysiol 1997; 20: 153-157.

6. Hsu W, Lin Y, Lang DJ, et al. Improved internal defibrillation success with shocks timed to the morphology electrogram. Circulation 1998; 98: 808-812.

7. Hsu W, Lin Y, Lang DJ, et al. Shock timing lowers transvenous defibrillation energy requirement. $J$ Electrcardiol 1998; 31 Suppl: 35-40.

8. Pak HN, Liu YB, Hayashi H, et al. Synchronization of ventricular fibrillation with real time feedback pacing: implication to low-energy defibrillation. Am J Physiol Heart Circ Physiol 2003; 285: H2704-H2711.
9. Strohmenger HU, Lindner KH and Brown CG. Analysis of the ventricular fibrillation ECG signal amplitude and frequency parameters as predictors of countershock success in humans. Chest 1997; 111: 584-589.

10. Kuelz KW, Hsia PW, Wise RM, et al. Integration of absolute ventricular fibrillation voltage correlates with successful defibrillation. IEEE Trans Biomed Eng 1994; 41: 782-791.

11. Kidwai BJ, McIntyre A, Anderson J, et al. Optimization of transthoracic ventricular defibrillation - biphasic and triphasic shocks, waveform rounding and synchronised shock delivery. J Electrocardiol 2002; 35: 235-244.

12. Kerber RE, Martins JB, Kienzle MG, et al. Energy, current, and success in defibrillation and cardioversion: clinical studies using an automated impedance-based method of energy adjustment. Circulation 1988; 77: 1038-1046.

13. Dalzell GWN, Cunningham SR, Anderson J, et al. Electrode pad size, transthoracic impedance and success of external ventricular defibrillation. Am J Cardiol 1989; 64: 741-744.

14. KenKnight BH, Eyuboglu BM and Ideker RE. Impedance to defibrillation countershock; does an optimal impedance exist? Pacing Clin Electrophysiol 1995; 18: 2068-2087.

15. Valenzuela TD, Roe DJ, Cretin S, et al. Estimating effectiveness of cardiac arrest interventions. Circulation 1997; 96: 3308-3313. 